\title{
Internal fixation of closed tibial fractures for the management of sports injuries
}

\author{
A. Abdel-Salam FRCS, K. S. Eyres* FRCS and J. Cleary FRCS \\ Department of Orthopaedics, Huddersfield Royal Infirmary, UK, and *Department of Human Metabolism and \\ Clinical Biochemistry, University of Sheffield, UK
}

\begin{abstract}
From 1985 to 1988, 90 closed tibial fractures were prospectively studied to assess the morbidity of such injuries to sports people and how this can be influenced by treatment regimens. All fractures were sustained during rugby or football matches. After random selection, 45 fractures were openly reduced and internally fixed (group A), and 45 fractures were manipulated under general anaesthesia and a long-leg plaster applied (group B). For patients in group A, below-knee plasters were used for 3 to 4 weeks, with immediate weight-bearing when tolerated. A total of $44(98 \%)$ tibiae united clinically and radiologically within 14-18 weeks. Within 4 months of surgery $28(62 \%)$ patients had returned to work and were able to play again by the following season. By 6 months 17 $(38 \%)$ patients had resumed normal activities. In group B, only $24(53 \%)$ tibiae united in a mean time of 16 weeks (range 12-40 weeks). Six (13\%) patients required bone grafting for non-union, and one patient subsequently developed osteomyelitis. Only ten $(22 \%)$ patients were back to work and playing sport again within 6 months of initial injury. It is concluded that internal fixation of closed tibial fractures as a primary procedure following low velocity sports injuries can be safely performed. It leads to a faster return to normal activities with fewer complications than does conservative treatment in plaster using contemporary methods.
\end{abstract}

Keywords: Tibial fracture, internal fixation, external fixation, sports injuries

\section{Introduction}

The role of internal fixation in the management of closed tibial fractures remains controversial. Most centres in the UK favour conservative methods and reserve operative techniques for those cases that have failed with these treatments. Although conservative management is often appropriate and avoids the potential hazards of internal fixation, the time spent in plaster and associated complications can be unacceptable, especially to sports people intent on returning to normal function as soon as possible.

A prospective study is presented to illustrate the results of open reduction and internal fixation

Address for correspondence: Dr K. S. Eyres, Department of Human Metabolism and Clinical Biochemistry, University of Sheffield, Beech Hill Road, Sheffield, UK

(C) 1991 Butterworth-Heinemann Ltd

0306-3674/91/040213-05 compared with the conservative treatment of closed tibial fractures. In particular, the time for restoration of normal activity and the complications of both methods are studied. The authors propose that preservation of the fracture haematoma is important for bone healing and a technique of internal fixation without fracture compression is described.

\section{Patients and methods}

Over the period 1985-1988, 90 cases of closed fractures of the tibia matched for age, sex and mode of injury were prospectively studied. Patients were randomly allocated to open reduction and internal fixation (group A), or manipulation under anaesthesia and the application of a long-leg plaster (group B). Random selection for treatment methods was made by card selection at presentation to the ward.

All fractures were operated upon by one of the authors (A.A.S.). Those treated surgically, nominated as group A, consisted of 42 men and three women, of mean age 27.4 (range 18-44) years. In group $B$ there were 41 men and four women, of mean age 28.7 (range 18-46) years (Table 1).

All injuries were sustained during rugby or football tackles (Table 2). In group A there were 25 oblique and

Table 1. Age and sex distribution and fracture distribution

\begin{tabular}{lcc}
\hline & Group A & Group B \\
\hline No. of patients & 45 & 45 \\
Men & 42 & 41 \\
Women & 3 & 4 \\
Mean(range) age(years) & $27.4(18-44)$ & $28.7(18-46)$ \\
No. of fractures & 45 & 45 \\
Right & 24 & 28 \\
Left & 21 & 17 \\
\hline
\end{tabular}

Table 2. Aetiology of the fractures

\begin{tabular}{lll}
\hline Mode of injury & Group $A$ & Group B \\
\hline Football injury & $35(77.8)$ & $33(73.3)$ \\
Rugby injury & $10(22.2)$ & $12(26.7)$ \\
\hline
\end{tabular}

Values in parentheses are percentages 
20 transverse fractures. Group B consisted of 23 oblique and 22 transverse fractures. The site of fracture is shown in Table 3. All patients were independently and critically assessed after both forms of treatment by an orthopaedic surgeon not involved in the primary care of the patients (K.S.E.).

\section{Criteria for inclusion}

All fractures were closed injuries with good quality overlying skin. Patients were excluded from the study unless these criteria were followed. If the position of the fracture could not be maintained by plaster alone, i.e. if more than $10^{\circ}$ of angulation in any plane or more than $1 \mathrm{~cm}$ of shortening persisted despite remanipulation or plaster wedging, then internal fixation was performed and these patients were also excluded from the study.

\section{Treatment}

Group B patients were treated with the application of a long-leg plaster with or without manipulation under anaesthesia as necessary.

At outpatient review, the fracture position was carefully checked and callus formation assessed. If the fracture position could not be maintained, the fracture was remanipulated or the plaster was wedged. If these methods failed to maintain the fracture position within the criteria described above, then internal fixation was performed and the patient was excluded from the study.

Weight-bearing was encouraged where possible and plasters were reduced to patella-bearing casts as appropriate.

In group $A$, the policy for inclusion for internal fixation was good quality skin as for inclusion into either group. At operation, the fracture haematoma was left undisturbed and the reduction gently performed using bone holding forceps and traction.

The authors consider that the fracture haematoma plays an important role in bone healing and should be left undisturbed to create an environment favourable to union. Therefore, instead of compressing the fracture site, the bone ends were realigned with the haematoma intact, often leaving 1-2 $\mathrm{mm}$ between the fracture ends. Large fracture fragments were realigned if they impeded fracture reduction. A wide direct compression plate was used to fix the fracture, with at least four screws above and below the fracture (Figure 1). The wound was closed without a drain. No tourniquet was used throughout this study. The leg was placed in a below-knee plaster with a walking heel after $24-48 h$ at which stage the dressing was

Table 3. Types of fracture configuration

\begin{tabular}{lcc}
\hline Site and type of fracture & Group A & Group B \\
\hline Upper third & 6 & 7 \\
Middle third & 18 & 20 \\
Distal third & 21 & 18 \\
Oblique & 25 & 23 \\
Transverse & 20 & 22 \\
\hline
\end{tabular}

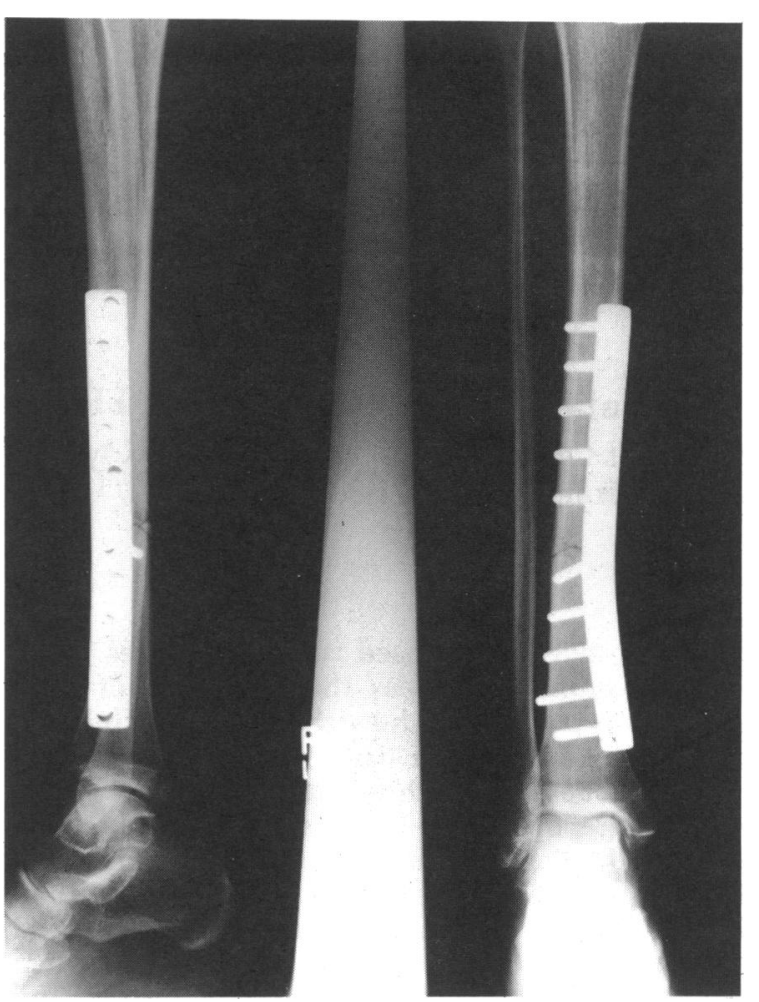

Figure 1. With maintenance of the fracture haematoma the heavy duty plate is applied. A slight gap can be seen

reduced. Patients were allowed home on crutches and advised to mobilize partial to full weight-bearing as tolerated. After 3-4 weeks patients were reviewed and plaster bivalved at which stage the sutures were removed and mobilization continued using support as required.

\section{Results}

In group A, all patients achieved union as judged by the ability to bear weight without pain, and the radiographic appearance of callus formation (Figure 2). Only one patient required reapplication of plaster owing to pain on walking 6 weeks after initial treatment. This patient achieved union at 25 weeks. In total, 28 patients had returned to normal activities/ work within 4 months of surgery.

Most patients in group A missed, on average, only one season of sports and all players could return to play. Conversely, the mean number of missed seasons for those patients treated conservatively was 3.5 and six players could not resume their sporting activities during the period of this study.

There were two complications in the 45 patients (Table 4) and both these patients presented 10 days after surgery with an erythematous wound. Wound swabs were negative and white blood counts and erythrocyte sedimentation rates were normal. In both cases the inflammation settled with bedrest, elevation and antibiotics. There were no patients with ankle or knee stiffness as a result of the early mobilization and none with malalignment or shortening. Subsequently 18 patients underwent plate removal (Figure 3). 
Internal fixation of closed tibial fractures: A. Abdel-Salam et al.
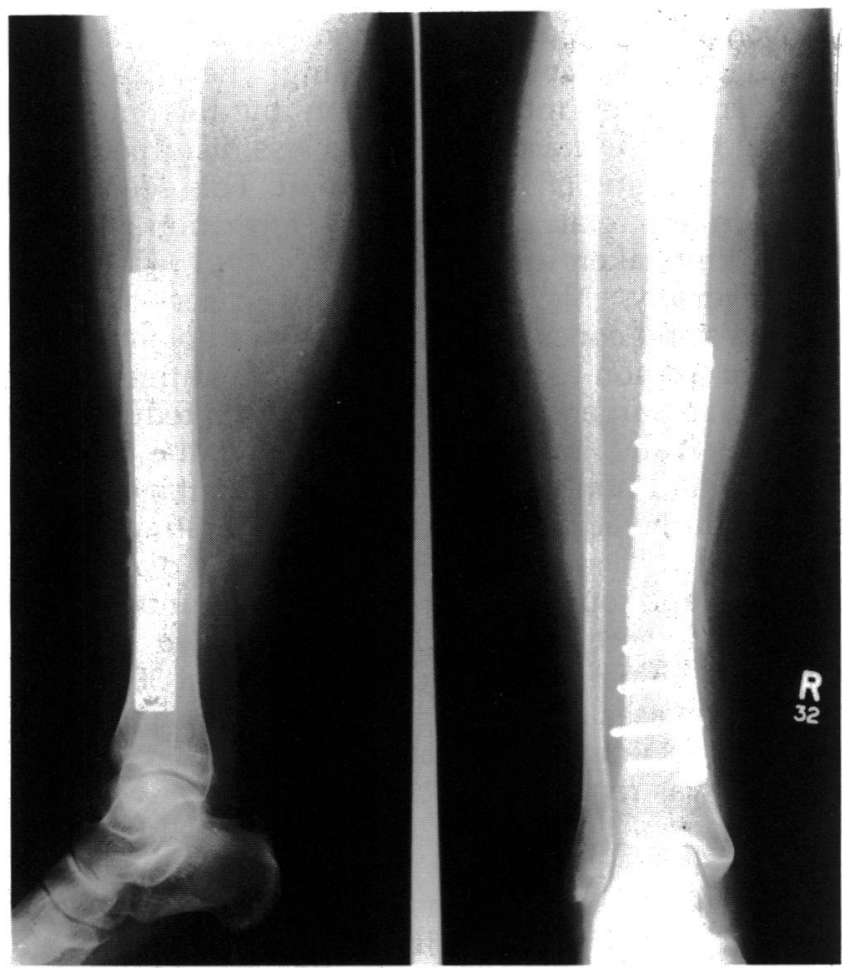

Figure 2. At 3 weeks the fracture gap has filled in and external callus is visible

In group $B$, however, only ten patients were back to work within 6 months. Tables 5 and 6 show the time spent in plaster. There were eight patients who required 30-40 weeks before union occurred.

The rate of complications in group B was higher, as shown in Table 7. Non-union occurred in six patients who required bone grafting. One of these patients
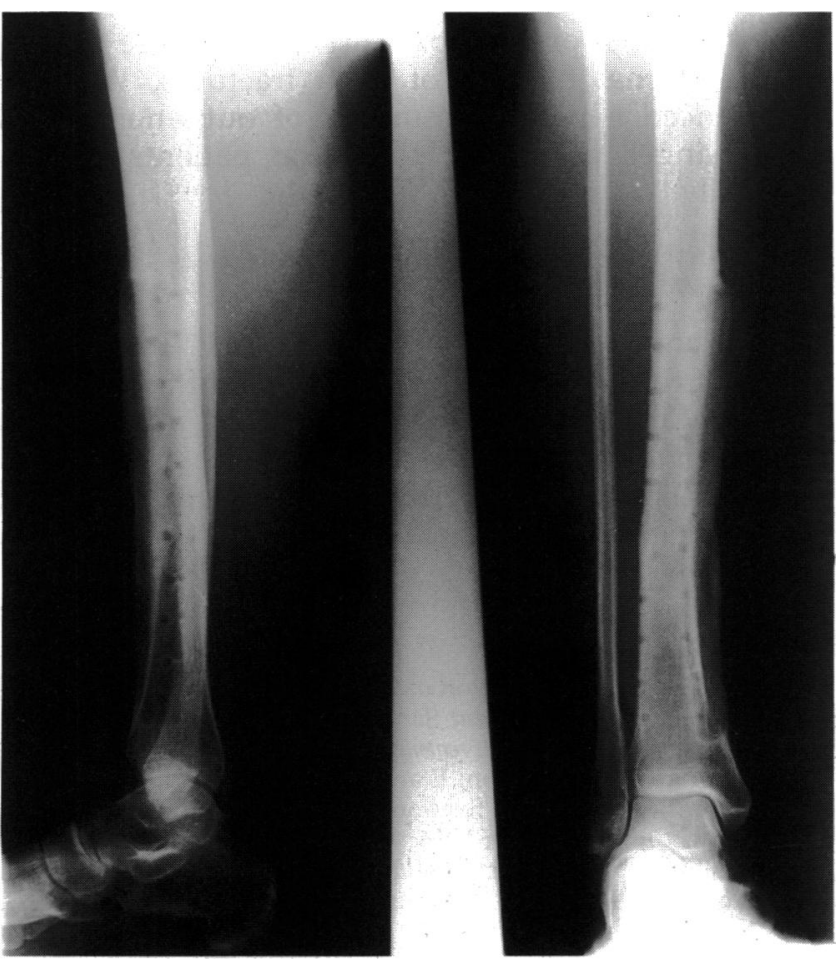

Figure 3. At 1 year after surgery the plate has been removed. Note good alignment without osteoporosis
Table 4. Complications seen in the surgically treated group (group A) after critical assessment by an independent observer

\begin{tabular}{ll}
\hline Complications & No. \\
\hline Superficial wound inflammation & 2 \\
Delayed union (united at 25 weeks) & 1 \\
Ankle stiffness & 0 \\
Knee stiffness (loss of $>15^{\circ}$ full extension or flexion) & 0 \\
Ankle pain & 0 \\
Leg shortening & 0 \\
Malalignment & 0 \\
\hline
\end{tabular}

Table 5. Time spent in plaster for those treated conservatively (group B)

\begin{tabular}{lc}
\hline Time in plaster (weeks) & No. of patients \\
\hline 12 & 4 \\
16 & 20 \\
20 & 9 \\
$28-30$ & 4 \\
$31-40$ & 8 \\
\hline
\end{tabular}

Ten patients went back to work in 6 months

Table 6. Time spent in plaster for patients treated surgically (group A)

\begin{tabular}{lc}
\hline Time in plaster (weeks) & No. of patients \\
\hline $3-4$ & 42 \\
$6-8$ & 3 \\
\hline
\end{tabular}

28 patients went back to work in 4 months, 17 patients went back to work in 6 months, 18 patients underwent plate removal

Table 7. Complications seen in the conservatively treated group (group B)

Complications

No.

Non-union requiring bone grafting

Osteomyelitis

Varus at fracture site

Valgus at fracture site

Shortening (of $2.5 \mathrm{~cm}$ )

Ankle osteoporosis and pain

Knee stiffness (loss of $>20^{\circ}$ full flexion or extension)

Ankle or subtalar stiffness

Persistent equinovarus foot deformity

Clawed toe from compartment syndrome

subsequently developed osteomyelitis which became chronic.

Following the outpatient review, five patients required remanipulation of the fracture because of loss of position, and seven patients required plaster wedging to correct deformity at the fracture site. In two patients the position of the fracture could not be maintained satisfactorily by these means and required internal fixation. These patients were excluded from the study. Despite this close observation during management, varus deformity of $10^{\circ}$ or more 
in two patients, and valgus deformity in four patients, subsequently occurred as the fracture went onto union (Figure 4). A total of ten patients had marked knee stiffness after plaster removal, although this predominantly occurred when Sarmiento plasters were not used. All patients in group B suffered variable degrees of ankle stiffness which required physiotherapy. One patient was found to have clawing of the toes after plaster removal, suggesting an undiagnosed compartment syndrome.

Using $\chi^{2}$ analysis and Yates' correction for continuity, the patients in group A regained normal activity and were able to return to work significantly faster than group B patients (mean 4 months and 8.2 months respectively, $P<0.005)$. The complication rate was also lower in those patients who underwent internal fixation $(P<0.001)$.

\section{Discussion}

Conflicting reports of the advantages and disadvantages of internal fixation as a mode of treatment for tibial fractures can be found in the literature ${ }^{1-5}$. Most surgeons in the UK, however, favour conservative methods such as manipulation and plaster or the application of external fixator systems.

Several authors advocate surgical treatment using plates, intramedullary devices or external fixator systems. Solheim ${ }^{6}$ treated 100 tibial fractures by the AO (Association for the Study of the Problems of Internal Fixation) method and encountered one case of non-union and four cases of deep infection. There were six cases of refracture. He concluded that the AO method was a definite advance in the management of tibial fracture. This had been previously advocated by Muller ${ }^{3}$.

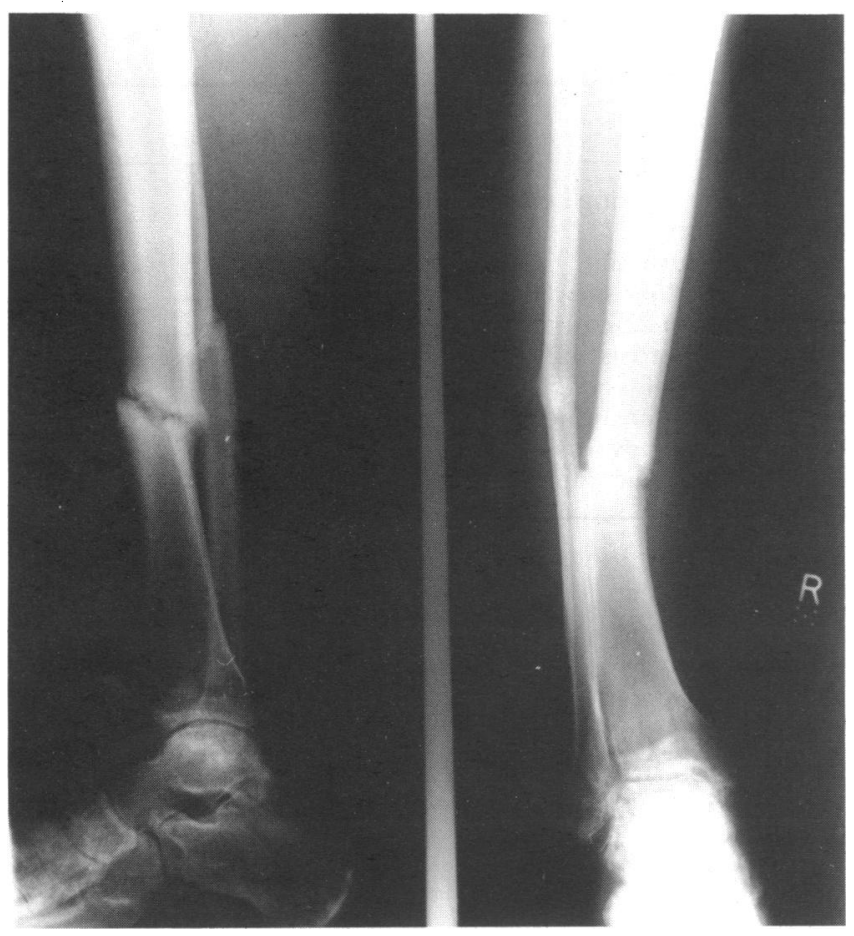

Figure 4. Patient treated conservatively illustrates deformity and non-union at 25 weeks after injury
Zucman and Maurer ${ }^{7}$ reported 346 tibial fractures managed by intramedullary nailing. They supported the benefits of internal fixation by this method, stating that the fracture site was less disturbed than by other means of internal fixation. This seemed to result in less retardation of callus formation relevant in the time taken for bone union ${ }^{8}$.

Conversely, Nicoll studied 705 cases of tibial fractures and concluded that there was not a case for internal fixation as a primary mode of treatment ${ }^{9}$. He stated that surgery was only justified if it reduced the deformity and joint stiffness, lowered the risks of delayed and non-union, and if the advantages could outweigh the additional hazards of operating.

Oni et al. ${ }^{10}$ studied 100 closed tibial diaphyseal fractures and found that 19 cases had not united by 20 weeks, the accepted definition for delayed union ${ }^{11}$. Of these, 15 patients did achieve union at 30 weeks. Only four cases required surgery because of failure to progress to union, so it was concluded that internal fixation was rarely justified.

Dehne et al. ${ }^{11}$ reported 207 cases of tibial fractures which had been treated by a close fitting cast with the knee held in full extension. The patients were allowed to bear weight immediately. The average time for union was 5 months, although the fracture types were not classified and patient ages were not stated.

In our series, the merits of internal fixation were compared with those of conservative treatment with plaster using contemporary techniques. The criteria for an adequate reduction were decided before starting the study in order to avoid the indiscriminate use of closed methods which would bias our results.

Notwithstanding, internal fixation of closed tibial fractures as a primary treatment is a safe and beneficial mode of management in a selected group of patients. Restoration of normal activities is especially important in sports people and we therefore recommend internal fixation of such fractures. We also experienced a reduced number of outpatient visits and changes of plaster for those patients treated surgically.

\section{Acknowledgements}

The authors wish to thank Professor L. Klenerman (Head of the Orthopaedic Department of the University of Liverpool Hospitals) for his advice and correction of this manuscript. Thanks also to the Medical Illustration Department of the Northern General Hospital, Sheffield.

\section{References}

1 Burwell HN. Plate fixation of tibial shaft fractures. A survey of 181 fractures. J Bone Joint Surg [Am]1971; 12: 386-93.

2 Charnley J. The Closed Treatment of Common Fractures, 3rd edn. Edinburgh and London: E and S Livingstone, 1961.

3 Muller ME. Internal fixation for fresh fractures and nonunion. J R Soc Med 1963; 56: 455-60.

4 Sarmiento A. A functional below-the-knee brace for tibial fractures. J Bone Joint Surg [Am] 1970; 52: 295-311.

5 Watson-Jones R, Coctart WD. Slow union of fractures with a study of 804 fractures. Br J Surg 1943; 30: 260-2.

6 Solheim K. Tibial fractures treated according to the AO method. Injury 1972; 4: 213-20. 
Internal fixation of closed tibial fractures: A. Abdel-Salam et al.

7 Zucman J, Maurer P. Primary medullary nailing of the tibia for fractures of the shaft in adults. Injury 1970; 2: 84-92

8 Minns RJ. Biomechanical study of internal fixation of the tibial shaft. I Biomech 1977; 10: 569-79.

9 Nicoll EA. Fractures of the tibial shaft; a survey of 705 cases. J Bone Joint Surg [Br] 1964; 46: 373-87.
10 Oni OO, Hun A, Gregg PJ. Healing of closed tibial fractures. I Bone Joint Surg [Br] 1988; 70: 787-90.

11 Dehne E, Deffer PA, Hall RM, Brown PW, Johnson EV. The natural history of the fractured tibia. Surg Clin North Am 1961; 41: 1495-513. 